\title{
A phase III, randomized, clinical, crossover study to evaluate the non-inferiority of an ophthalmic solution of preservative-free latanoprost at $0.005 \%$ in patients with primary open-angle glaucoma
}

\author{
Victoria E Sánchez-Castellanos ${ }^{1}$, José A Paczka ${ }^{2,3,4}$, Francisco Gómez-Aguayo ${ }^{5}$, Felix Gil-Carrasco ${ }^{6}$, Curt Hartleben ${ }^{7}$, Leopoldo Baiza- \\ Durán $^{8}$, Oscar Olvera-Montaño ${ }^{8}$, Alejandra Sánchez-Rios ${ }^{8}$ and Patricia Muñoz-Villegas ${ }^{8 *}$ \\ ${ }^{1}$ Aris Vision Institute, Av. México 3217, Guadalajara, Jalisco, Mexico \\ ${ }^{2}$ Global Glaucoma Institute, Mariano Azuela 37 P.B., 44600, Guadalajara, Jalisco, Mexico \\ ${ }^{3}$ Unidad de Diagnóstico Temprano del Glaucoma, Boulevard Puerta de Hierro 5150, Int. 404-A, Zapopan, Jalisco, Mexico \\ ${ }^{4}$ Instituto de Oftalmología y Ciencias Visuales, CUCS, Universidad de Guadalajara, Sierra Mojada 950, E-N, Guadalajara, Jalisco, Mexico \\ ${ }^{5}$ Private Practice, Eulogio Parra 3061, Guadalajara, Jalisco, Mexico \\ ${ }^{6}$ Asociación para evitar la ceguera: APEC, Vicente García Torres 46, Mexico City, Mexico \\ ${ }^{7}$ Fundación de Asistencia Privada Conde de Valenciana I.A.P., Chimalpopoca 14, Mexico City, Mexico \\ ${ }^{8}$ Laboratorios Sophia, S.A. de C.V. Paseo del Norte 5255, Zapopan, Jalisco, Mexico
}

\begin{abstract}
Purpose: The aim of this crossover study was to evaluate the non-inferiority of PRO-067 (ophthalmic solution of preservative-free latanoprost at 0.005\%) against a formulation of latanoprost at $0.005 \%$ containing a preservative (GOF) in respect to controlling the intraocular pressure (IOP) of patients with primary open-angle glaucoma (POAG), previously treated and clinically controlled. Safety and tolerability were also assessed by the incidence of adverse events (AEs), ocular findings, the VF-14 index, and a questionnaire on ocular comfort.

Methods: This was a randomized, prospective, multicenter, double-blind, crossover (AB:BA) clinical trial including POAG patients previously treated and clinically controlled for at least two months with a solution of latanoprost at $0.005 \%$ containing a preservative. A total of 99 patients were randomly-assigned to one of two sequences: sequence 1: PRO-067 $\rightarrow$ GOF ( $n=49)$; sequence 2: GOF $\rightarrow$ PRO-067 ( $n=50)$.

Results: PRO-067 was found to be effective in controlling and maintaining IOP under control during the study. The safety of the two medications was similar, as no serious or severe AEs were reported in association with either drug. The tolerability of the two medications - evaluated by ocular findings, the VF-14 index and a questionnaire of ocular comfort- was also determined to be similar.

Conclusion: These findings suggest that the usage of the preservative-free latanoprost at $0.005 \%$ is as effective as the formulation of latanoprost at $0.005 \%$ with the preservative. Finally, treatment with PRO-067 showed safety and tolerability issues similar to those of latanoprost at $0.005 \%$ with a preservative.
\end{abstract}

\begin{abstract}
Abbreviations
PGAs: Prostaglandin Analogues; BAK: Benzalkonium Chloride; AEs: Adverse Events; POAG: Primary Open-Angle Glaucoma; GOF: GAAP Ofteno; OHT: Intraocular Hypertension; OSD: Ocular Surface Disease; IOP: Intraocular Pressure; SFB: Sensation of Foreign Body; TBUT: Tear Break-Up Time.

\section{Introduction}

Glaucoma is a progressive optic neuropathy characterized by the loss of retinal ganglion cells. This condition gives the optic disc a distinctive appearance and results in a concomitant loss of visual function [1]. This silent illness is the second-leading cause of blindness worldwide, and the most common cause of irreversible blindness in adult populations [2]. Primary open-angle glaucoma (POAG) is the most prevalent form of this condition. Prostaglandin analogues (PGA) are the most effective
\end{abstract}

hypotensive ocular drugs and the most frequently prescribed to treat POAG. Although the action mechanism of this pharmacological class is not yet completely clear, it seems that an increased aqueous humor drainage through the uveoscleral and, to a lesser degree, the trabecular meshwork is crucial to induce a reduction of IOP [3].

Prostaglandins are normally found in ocular tissues, and they modulate a broad spectrum of biological processes and responses,

${ }^{\star}$ Correspondence to: Muñoz-Villegas P, Laboratorios Sophia, S.A. de C.V., Paseo del Norte 5255, Guadalajara Technology Park, Zapopan, Jalisco, Mexico, 45010; Tel: +52 33014200-1018; E-mail: patricia.munoz@sophia.com.mx

Key words: primary open-angle glaucoma, latanoprost, preservative-free formulation, intraocular pressure

Received: May 15, 2018; Accepted: May 23, 2018; Published: May 29, 2018 
either alone or in combination with autacoids. The esterification of carboxylic acid in a PGF2 $\alpha$ reduces the amount of medication required to decrease intraocular pressure (IOP) and so acts to reduce the side effects associated with the PGA [4,5].

Latanoprost is a PGA ester, a selective agonist of the FP subtype prostanoid receptor. Just one drop of latanoprost at $0.005 \%$ per day has been shown to have good efficacy in reducing IOP in POAG patients [3]. The first ophthalmic formulation of $0.005 \%$ latanoprost marketed was Xalatan (Pfizer, Ltd., NY, USA). GAAP Ofteno (Laboratorios Sophia, S.A. de C.V., Jalisco, Mexico) is also a $0.005 \%$ latanoprost formulation, but with an important innovation: it is stable at different temperatures while maintaining the efficacy and safety characteristics of Xalatan [6].

In controlled clinical trials involving patients with POAG or intraocular hypertension (OHT), monotherapy with $0.005 \%$ latanoprost reduced IOP by $22-39 \%$ during $1-12$ months of treatment. Also, IOP remained stable in patients who received the drug for $1-2$ years $[3,7]$.

Because most patients with glaucoma require long-term medical treatment to control IOP, monitoring tolerability is important when it comes to selecting a drug. The most common treatment for glaucoma consists in combining two drugs, but this polypharmaceutical approach may be inconvenient for patients and so result in poor adherence to treatment. PGAs are the drops most often used to treat glaucoma because of their well-known safety profile and simple scheme of administration, which requires only one application per day.

Benzalkonium chloride (BAK) is the preservative most often used in ophthalmological preparations. It is also a strong antibacterial agent [8]. BAK-free PGAs present few toxic effects on the ocular surface, and so might improve patients' adherence to treatment and, therefore, overall success rates. BAK has been correlate with inflammation, conjunctival and corneal damage, decrease in the stability of the tear film because it contains a compound of polyquaternary ammonium that affects the cell membranes and the lipid component of the tears, resulting in dry eyes and irritation [8-11]. Given this background, treating POAG or OHT patients with a minimum of BAK-free medications should not only reduce IOP, but also decrease the appearance of the symptomatology of ocular surface disease (OSD) [12-15].

Finally, in crossover designs, each subject receives all treatments that are being investigated, but at different times, the order in which subject receives the treatments is randomized. Utilizing a crossover design, for the right patient population and treatment minimizes the impact of confounding covariables and allows each subject to act as her or his own comparison. In theory, a crossover design can achieve the same precision as a trial with parallel groups but requires only half the sample size. Therefore, it can facilitate determining the real efficacy of two treatments [16].

In light of this, the objective of the present study was to evaluate the non-inferiority of PRO-067, a preservative-free ophthalmic solution, compared to GOF in maintaining the IOP of POAG patients previously treated and controlled with that medication. The parameter of efficacy consisted in maintaining IOP below the target value. Tolerability and safety were assessed by means of a questionnaire on ocular comfort, the VF-14 index, ocular findings, and the incidence of adverse events (AEs).

\section{Methods}

\section{Study design}

This was a 2-month, phase III, randomized, prospective, doubleblind, multicenter, crossover AB:BA, clinical trial (ClinicalTrials.gov registration number: NCT02801617), conducted in seven centers in
Mexico (see the list in the Acknowledgments section). It complied with the principles of the Declaration of Helsinki and followed the standards of Good Clinical Practices as well as all applicable Mexican regulations. A letter of informed consent was read, signed and dated by all patients before performing any procedures of the study protocol. Patients were recruited between October 2015 and July 2017.

\section{Participants}

The patients recruited were $\geq 18$ and $\leq 90$ years old with diagnoses of POAG who had used GOF for at least two months before inclusion and had IOP equal to or below the target IOP, according to the treating ophthalmologist's criteria. They were classified as having slight, moderate or severe glaucomatous damage (stage 1 to 4 according to Hodapp-Parrish-Anderson criteria, [17]). In neither sequence were patients allowed a washing period between applying the different medications (PRO-067, GOF)

The exclusion criteria were blindness in one eye, best-corrected visual acuity of 20/200, or worse, in one or both eyes, loss of visual field indicative of end-stage glaucoma, clinical histories that included untreated narrow-angle glaucoma with total or partial closure of the angle of either eye, previous cataract surgery or any other intraocular intervention within the 6 months prior to the beginning of the study, the use of other medications with potentially significant effects on IOP, and pregnancy, risk of pregnancy, or lactation.

The principle variable for determining efficacy was maintaining IOP within the target range during the period between the initial (baseline) and final visits.

\section{Treatment and evaluation}

A total of 116 patients were evaluated for their eligibility during an assessment visit held 6-8 weeks before the initial visit. At that time, their clinical histories were documented, and an ocular examination was performed. If the patient satisfied all the inclusion criteria and none of the exclusion criteria, she/he was included in the study. The 2-month study period included 5 programmed visits: initial (day 1 ), safety 1 (day 15), crossover (day 30), safety 2 (day 45), and final (day 60). On each occasion, the IOP of each eye was measured (using a calibrated Goldmann applanation tonometer). Best-corrected visual acuity was also tested, using the Snellen chart, while visual fields were determined by Humphrey automated perimetry, applying the SITA-Standard 24-2 strategy (Carl Zeiss Meditec AG, Dublin, California, USA). The ocular surface was evaluated with a slit lamp aided by fluorescein staining. Surface dye staining was classified as slight, moderate or severe per the percentage of the affected area. Finally, a questionnaire of ocular comfort and the VF-14 index were applied during the initial, crossover (day 30) and final visits (day 60).

During the initial visit, the patients were assigned randomly and simply (1:1) to sequence 1 or 2 . To keep the treatment masked, the bottles with the study drugs bore identical labels. On the initial visit, the patients in sequence $1(n=49)$ received (day 1) PRO-067 (BAKfree latanoprost at $0.005 \%$; multidose-low density polyethylene bottle, closure system of high density polyethylene with a silicone valve system, which allow to preserve the sterile solution without preservatives), while those in sequence $2(\mathrm{n}=50)$ received GAAP Ofteno (GOF). On the crossover visit after the initial 1-month period (day 30 ), the patients in sequence 1 were given GOF, and those in sequence 2 began to use PRO-067. The researchers, patients and other personnel involved in the study were masked to treatment assignation during the protocol Patients were instructed to apply one drop of the drug in each eye daily. A safety visit was made two weeks after treatment period 2 (i.e., day 75). 
In the event of pregnancy or the occurrence of a serious adverse event, or if a patient wished to cease participating in the study, or for safety reasons determined by the researchers, she/he was allowed to withdraw. Adverse events were carefully monitored throughout the study. Figure 1 presents a flow chart of participants.

\section{Variables}

Efficacy was evaluated by monitoring IOP during the time between the initial and final visits (day 60). The variables used to analyze tolerability and safety were: ocular findings (tear break-up time, conjunctival hyperemia, chemosis, sensation of a foreign body, tearing, or a burning sensation), the VF-14 index, the questionnaire on ocular comfort, and the incidence of adverse events.

\section{Statistics}

Data on safety were analyzed for the to-be-treated population (ITT), while data on efficacy and tolerability were analyzed for the protocol population (PP). The latter was defined as each patient assigned who showed no marked deviation from the protocol. The calculation of the sample size was based on the non-inferiority criteria of PRO-067 and GOF. It was determined that at least 41 patients per group were required to detect a difference of at least $2.0 \mathrm{mmHg}$ in maintaining mean IOP between treatments using a significance level of 0.05 , with a potency of 0.80 . After adjusting for the dropout rate, it was deemed necessary to assign a total of 100 patients.

Data were analyzed using analysis of variance (ANOVA) and a Student's $t$ test for repeated measures. In the case of multiple comparisons, Bonferroni correction was applied to adjust the $p$ value for individual points. All the categorical variables were analyzed with Pearson's Chi square or Fisher's exact test. All $p$ values presented are two-tailed, considering $\mathrm{p} \leq 0.05$ as significant. Analyses were performed with SPSS version 19.0 software (SPSS Inc., Chicago, IL, USA).

\section{Results}

\section{Subjects' characteristics}

A total of 116 patients were evaluated for their possible participation in the study, and 114 of them were assigned to one of the sequences. Seven patients were lost during follow-up. The remaining population (ITT) thus consisted of 107 patients, but data from 8 of them were excluded from the final analysis due to lack of adherence to treatment (below 80\%). As a result, the protocol population (PP) was 99 patients. Their mean age \pm standard deviation was $63.2 \pm 10.7$ years (range: 24 $84) ; 78.8 \%$ of them were women. Mean best-corrected visual acuity (SD) was 0.1 (0.12) (in LogMAR) for both sequences. Humphrey automated perimetry generated a mean deviation (DM) of $-5.1 \pm 5.6 \mathrm{~dB}$ for sequence 1 , and of $-3.7 \pm 3.9 \mathrm{~dB}$ for sequence 2. No differences were observed in the demographic characteristics of the patients assigned (Patients' demographic data are shown in Table 1).

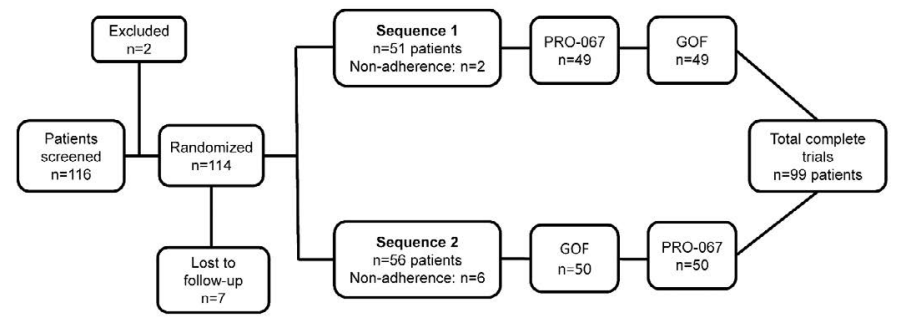

Figure 1. Diagram of the patients enrolled in the study
Table 1. Baseline characteristics ( $\mathrm{n}=99$ completed patients).

\begin{tabular}{|c|c|c|c|}
\hline & Sequence $1(n=49)$ & Sequence $2(n=50)$ & \\
\hline & PRO-067 $\rightarrow$ GOF & $\mathrm{GOF} \rightarrow \mathrm{PRO}-067$ & $p$ \\
\hline \multicolumn{3}{|l|}{ Gender, n (\%) } & \multirow{3}{*}{0.063} \\
\hline Female & $35(71.4)$ & $43(86)$ & \\
\hline Male & $14(28.6)$ & $7(14)$ & \\
\hline Mean Age (SD), years & $63.5(9.7)$ & $62.9(11.8)$ & 0.794 \\
\hline Mean deviation (SD), $\mathrm{dB}$ & $-5.1(5.6)$ & $-3.7(3.9)$ & 0.160 \\
\hline Corneal Pachymetry (SD), $\boldsymbol{\mu} \mathrm{m}$ & $532.9(27.6)$ & $542.5(34.4)$ & 0.128 \\
\hline TBUT (SD), seconds & $7.5(2.4)$ & $7.2(2.3)$ & 0.467 \\
\hline $\begin{array}{l}\text { Mean Snellen 20/ best-corrected } \\
\text { visual acuity }\end{array}$ & $20(10)$ & $30(10)$ & 0.243 \\
\hline Mean baseline IOP (SD), $\mathrm{mmHg}$ & $14.2(2.4)$ & $14.0(2.4)$ & 0.657 \\
\hline
\end{tabular}

TBUT: Tear break up time; IOP: Intraocular pressure; SD: standard deviation; $X^{2}$ Fisher's exact test; $t$-test unpaired sample.

\section{Efficacy}

Intraocular pressure: IOP was analyzed in 99 patients, 49 of them assigned to sequence 1 (PRO-067: days 1-30, GOF days 31-60), and 50 to sequence 2 (GOF: days 1-30, PRO-067: days 31-60). Both products contain latanoprost at $0.005 \%$, but $\mathrm{PRO}-067$ is BAK-free. To analyze efficacy in terms of the non-inferiority of PRO-067 vs. GOF, maintenance of IOP was analyzed before and after use of both drugs. The study's crossover design facilitates determining the real efficacy of the two treatments (Table 2).

In sequence 1 , the mean \pm standard deviation of baseline IOP was $14.2 \pm 2.4$. After using PRO-067, it was $13.8 \pm 2.1 \mathrm{mmHg}$; indicating a reduction of $0.40 \mathrm{mmHg}$ with respect to the initial value. This difference was not significant $\left(\mathrm{t}_{(48)}=-1.731, p=0.090\right)$. At the end of period 2 of treatment, IOP was $14.0 \pm 2.5 \mathrm{mmHg}$; an increase of $0.20 \mathrm{mmHg}$ over the period with PRO-067. This difference was not significant either $\left(\mathrm{t}_{(48)}=0.718, p=0.476\right)$. The patients in sequence one thus experienced a decrease of $0.20 \mathrm{mmHg}$ by the end of the study. This difference was not significant with respect to the initial value $\left(\mathrm{t}_{(48)}=-1.083, p=0.284\right)$.

For sequence 2, baseline IOP was $14.0 \pm 2.4 \mathrm{mmHg}$. At the end of the first period, it was $13.9 \pm 2.2 \mathrm{mmHg}$; that is, $0.10 \mathrm{mmHg}$ below initial IOP, but not a significant change $\left(\mathrm{t}_{(49)}=-0.569, p=0.572\right)$. After use of PRO-067, IOP was $14.1 \pm 2.4$; i.e., an increase of $0.20 \mathrm{mmHg}$ compared to the use of GOF. Once again, the difference was not significant $\left(\mathrm{t}_{(49)}=\right.$ $0.614, p=0.542$ ). Thus, at the conclusion of the study, the patients in sequence 2 had a non-significant increase of $0.10 \mathrm{mmHg}$ with respect to their baseline IOP $\left(\mathrm{t}_{(48)}=0.139, p=0.890\right)$.

Therefore, control of IOP was achieved during the two months of the clinical protocol, regardless of the period and sequence of study $\left(\mathrm{F}_{(4,192)}=1.442, p=0.222\right)$. The efficacy in controlling IOP showed no statistical differences between the two groups $\left(\mathrm{F}_{(1,48)}=0.1675, p=0.898\right)$ (Figure 2).

\section{Safety}

Adverse events: data on safety were analyzed for the to-be-treated population (ITT), a total of 44 AEs were reported by $28.07 \%(32 / 114)$ of the subjects randomized during the study. There were no significant differences between treatments in the incidence of $\mathrm{AE}\left(X_{(1)}^{2}=1.104\right.$, $p=0.329)$, as 24 were reported for PRO-067 $-20.8 \%(5 / 24)$ slight and $79.2 \%(19 / 24)$ moderate- while for GOF, subjects reported 20 AEs: $35.0 \%(7 / 20)$ slight, and $65.0 \%(13 / 20)$ moderate. No serious adverse events occurred during the study. There were no significant differences in the severity of AEs between treatments $\left(X_{(1)}^{2}=1.104, p=0.329\right)$. 
Sánchez-Castellanos VE (2018) A phase III, randomized, clinical, crossover study to evaluate the non-inferiority of an ophthalmic solution of preservative-free latanoprost at $0.005 \%$ in patients with primary open-angle glaucoma

Table 2. Maintenance of intraocular pressure levels ( $\mathrm{mmHg})$.

\begin{tabular}{|c|c|c|c|c|}
\hline Visit & \multicolumn{2}{|c|}{ Mean IOP (95\% CI) } & $\begin{array}{c}\text { Mean adjusted } \\
\text { difference }\end{array}$ & $p$ \\
\hline & Sequence 1 & Sequence 2 & & \\
\hline 2 weeks & $13.8(-0.83,-0.03)$ & $13.9(-0.63,0.27)$ & -0.033 & 0.938 \\
\hline Crossover & $13.8(-0.38,0.42)$ & $13.9(-0.34,0.46)$ & -0.073 & 0.865 \\
\hline 6 weeks & $13.8(-0.40,0.25)$ & $13.9(-0.31,0.45)$ & -0.214 & 0.647 \\
\hline Final & $14.0(-0.17,0.60)$ & $14.1(-0.34,0.50)$ & -0.080 & 0.869 \\
\hline
\end{tabular}

Mean intraocular pressure (IOP) $(95 \% \mathrm{CI}$ for difference); $t$ test for repeated measures. Sequence 1: PRO-067 $\rightarrow$ GOF $(n=49)$. Sequence $2:$ GOF $\rightarrow$ PRO-067 $(n=50)$.

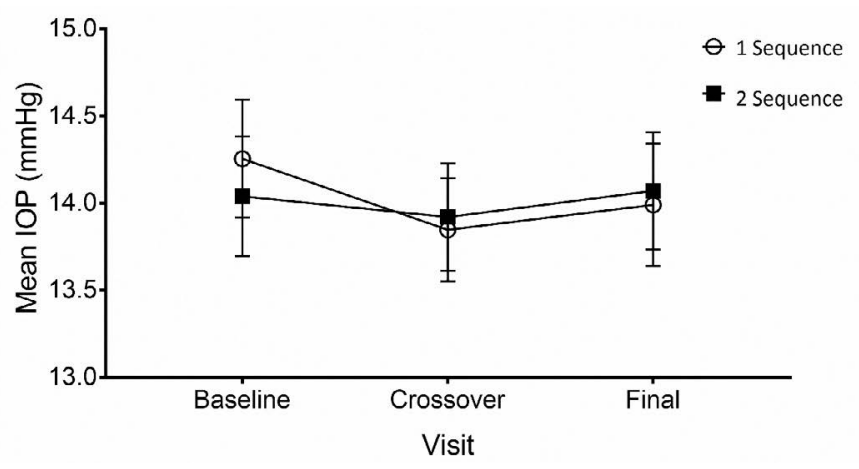

Figure 2. Mean intraocular pressure \pm S.E.M. at each experimental visit for sequences 1 (white circle) and 2 (black square). Sequence 1: PRO-067 days 1-30, GOF days 31 to end $(\mathrm{n}=49)$. Sequence 2 : GOF days 1-30, and PRO-067 days 31 to end $(n=50)$.

Non-ocular AEs constituted $75.0 \%$ of all cases. The most common non-ocular AEs reported was headache (9.1\%), whereas the most frequent ocular AEs was conjunctivitis (11.4\%), followed by subconjunctival hemorrhage (9.1\%). There were no significant differences in the type or frequency of AEs between treatments $\left(X^{2}{ }_{(5)}=5.197\right.$, $p=0.392$ ). The adverse events are shown in Table 3 .

\section{Tolerability}

Ocular findings: tear break-up time (TBUT), conjunctival hyperemia, chemosis, sensation of a foreign body (SFB), tearing, and a burning sensation, were considered the parameters of our ocular findings. For TBUT, no differences were observed between treatments for either sequence, and this factor remained constant throughout the 8-week study period, regardless of sequence. For conjunctival hyperemia, the grades found on the baseline visit were 0 (47), 1(46), $2(5)$ and 3 (1). On the crossover visit, grades 1 and 2 were seen in 45 and 2 patients, respectively, while on the final visit, the incidence of conjunctival hyperemia decreased to only 30 cases of grade 1 and 4 cases of grade 2, among all patients treated with one of the two medications. After the change from PRO-067 to GOF, and vice versa, no significant differences were observed in the frequency or severity of hyperemia, but a significant reduction was found on the final visit with respect to the initial one ( $X^{2}$ test, $p=0.039$ ) (Figure 3 ). In addition, no presence of chemosis was detected in either sequence or period. Finally, no significant differences were observed for the sensation of a foreign body, tearing, or burning between the sequences in each period of the protocol $(\mathrm{p}>0.05)$ (Table 4).

VF-14 index: The VF-14 visual function index is a questionnaire that evaluates the visual function in relation to daily-use vision in patients with cataracts, glaucoma and other ophthalmological diseases. Our participants graded their difficulty in performing everyday activities (e.g. reading small letters, driving at night, cooking, etc.). Each questionnaire was scored on a scale of 0-4. Average scores were then multiplied by 25 to give a scale of $0-100$. No differences were observed between the sequences during the clinical trial, on the baseline visit $\left(X_{(97)}^{2}=0.06, p=0.952\right)$, the crossover visit $\left(X_{(97)}^{2}=0.279\right.$, $p=0.781)$, or the final visit $\left(X^{2}{ }_{97)}=0.097, p=0.923\right)$. The average score (DE) after using PRO-067 was 87.6 (15.3) for sequence 1 and 86.7 (15.6) for sequence 2 , while after using GOF, it was 87.2 (16.5) for sequence 1 and 86.9 (16.4) for sequence 2 (Table 5).

Questionnaire on ocular comfort: participants were asked to score the discomfort they felt during the study on a scale of $0-10$, with 0 being 'no discomfort', and 10, 'unbearable discomfort'. The factors considered

Table 3. Safety analysis: Treatment-related adverse events ( $44 \mathrm{AE} / 32$ subjects).

\begin{tabular}{|l|c|c|c|}
\hline & \multicolumn{2}{|c|}{ Treatment Group } & \\
\hline & PRO-067 & GOF & Total \% \\
\hline AEs, n (\%) & $24(21.1)$ & $20(17.5)$ & \\
\hline Non-ocular, $n$ (\%) & $21(87.5)$ & $12(60.0)$ & 75.0 \\
\hline Rhinopharyngitis, n (\%) & $2(8.3)$ & $1(5.0)$ & 6.8 \\
\hline Headache, n (\%) & $3(12.5)$ & $1(5.0)$ & 9.1 \\
\hline Other, n (\%) & $16(66.7)$ & $10(50)$ & 59.1 \\
\hline & & & \\
\hline Ocular, $n$ (\%) & $3(12.5)$ & $8(40)$ & 25.0 \\
\hline Conjunctivitis, n (\%) & $1(4.2)$ & $4(20.0)$ & 11.4 \\
\hline Subconjunctival hemorrhage, n (\%) & $1(4.2)$ & $3(15.0)$ & 9.1 \\
\hline Other, n (\%) & $1(4.2)$ & $1(5.0)$ & 4.5 \\
\hline
\end{tabular}

AEs: Adverse events present per patient, data on safety were analyzed for the to-be-treated population (ITT), $\mathrm{n}=114$. No significant differences between groups, all $p$ values $\left(X^{2}\right.$ Pearson) were $>0.05$

Figure 3. Frequency and severity of hyperemia. Figures indicate the number of patients, ${ }^{*} \mathrm{p}<0.05$ for the number of patients with hyperemia compared to the baseline ( $X^{2}$ test). A. sequence 1: PRO-067 days 1-30, GOF days 31 to end $(n=49)$. B. Sequence 2: GOF days $1-30$, and PRO-067 days 31 to end $(\mathrm{n}=50)$.

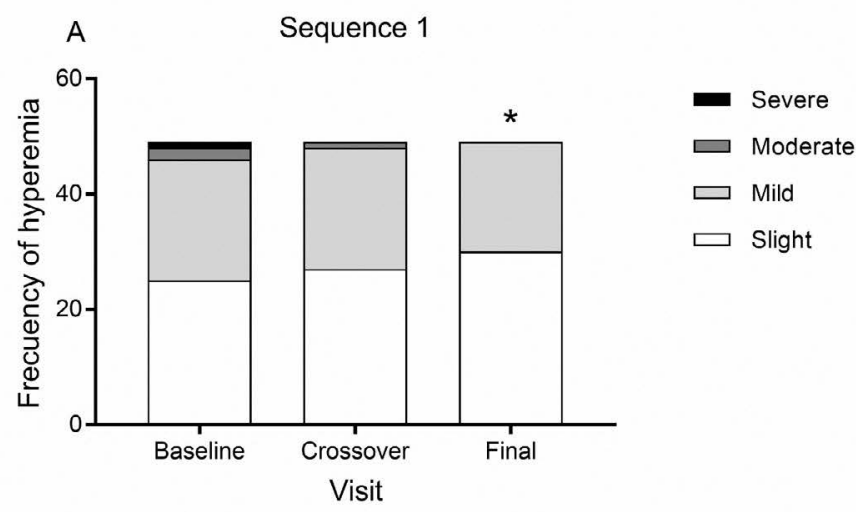

B

Sequence 2

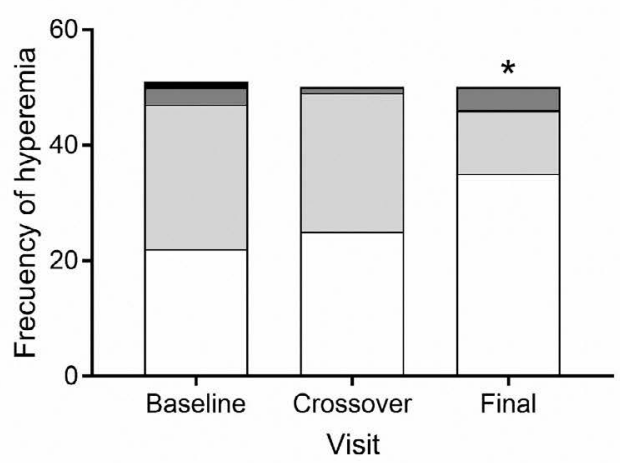


Table 4. Comparison of ocular findings between sequences 1 and 2

\begin{tabular}{|l|c|c|c|c|c|c|}
\hline & \multicolumn{2}{|c}{ Baseline } & \multicolumn{2}{c|}{ PRO-067 } & \multicolumn{2}{c|}{ GOF } \\
\hline Sequence & $\mathbf{1}$ & $\mathbf{2}$ & $\mathbf{1}$ & $\mathbf{2}$ & $\mathbf{1}$ & $\mathbf{2}$ \\
\hline Mean TBUT & $7.5(2.4)$ & $7.2(2.3)$ & $8.0(2.8)$ & $7.3(2.2)$ & $7.3(1.9)$ & $7.6(2.3)$ \\
\hline Hyperemia, n (\%) & $24(49.0)$ & $28(56.0)$ & $22(44.9)$ & $15(30.0)$ & $19(38.8)$ & $25(50.0)$ \\
\hline Chemosis, n (\%) & $0(0)$ & $0(0)$ & $0(0)$ & $0(0)$ & $0(0)$ & $0(0)$ \\
\hline SFB, n (\%) & $13(26.5)$ & $21(42.0)$ & $13(26.5)$ & $16(32.0)$ & $10(20.4)$ & $15(30.0)$ \\
\hline Tearing, n (\%) & $4(8.2)$ & $5(10.0)$ & $5(10.2)$ & $4(8.0)$ & $2(4.1)$ & $6(12.0)$ \\
\hline Burning, n (\%) & $11(22.4)$ & $11(22.0)$ & $7(14.3)$ & $9(18.0)$ & $5(10.2)$ & $12(24.0)$ \\
\hline
\end{tabular}

Sequence 1: PRO-067 $\rightarrow$ GOF $(n=49)$. Sequence 2: GOF $\rightarrow$ PRO-067 $(\mathrm{n}=50)$. SFB Sensation of foreign body, TBUT: Tear break-up time (seconds). Frequencies (\%), Pearson $X^{2}$ test and student $t$ test for independent groups. $p>0.05$ in all cases

Table 5. Results of the VF-14 index and the questionnaire on ocular comfort

\begin{tabular}{|c|c|c|c|c|c|c|}
\hline & \multicolumn{2}{|c|}{ Baseline } & \multicolumn{2}{c|}{ PRO-067 } & \multicolumn{2}{c|}{ GOF } \\
\hline Sequence & $\mathbf{1}$ & $\mathbf{2}$ & $\mathbf{1}$ & $\mathbf{2}$ & $\mathbf{1}$ & $\mathbf{2}$ \\
\hline VF-14 & $83.9(16.2)$ & $83.8(17.2)$ & $83.6(15.3)$ & $86.7(15.7)$ & $87.2(16.5)$ & $86.9(16.4)$ \\
\hline Fatigue & $0.5(1.5)$ & $0.7(1.6)$ & $0.7(1.7)$ & $0.4(1.3)$ & $0.3(0.7)$ & $0.5(1.4)$ \\
\hline Burning & $1.2(1.9)$ & $1.4(2.2)$ & $0.9(1.4)$ & $1.0(1.9)$ & $0.5(1.5)$ & $0.7(1.4)$ \\
\hline Itching & $0.5(1.4)$ & $2.0(2.6)^{*}$ & $0.4(1.0)$ & $1.1(1.8)^{*}$ & $0.2(0.6)$ & $0.8(1.4)^{*}$ \\
\hline Dryness & $1.5(1.8)$ & $1.8(2.2)$ & $1.2(1.5)$ & $1.3(2.0)$ & $0.9(1.6)$ & $0.9(1.3)$ \\
\hline Pain & $0.5(1.0)$ & $0.4(1.4)$ & $0.2(0.6)$ & $0.5(1.3)$ & $0.2(1.0)$ & $0.2(0.7)$ \\
\hline
\end{tabular}

Sequence 1: PRO-067 $\rightarrow$ GOF $(n=49)$. Sequence 2: GOF $\rightarrow$ PRO-067 $(n=50)$. Means (standard deviation). Student $t$ test for independent groups, ${ }^{*} p<0.05$

during the administration of each drug were: fatigue, burning, itching, dryness and pain. The scores reported for both sequences and both drugs were low. Only sequence 2 showed significant differences for the sensation of itching, compared to sequence 1 , basal $\left(\mathrm{t}_{(97)}=-3.344\right.$, $p=0.001)$, and GOF $\left(\mathrm{t}_{(97)}=-2.592, p=0.011\right)$. Although this result continued during use of PRO-067 $\left(\mathrm{t}_{(97)}=-2.428, p=0.017\right)$, the scores for the patients in this sequence were always below 5 (Table 5 ).

\section{Discussion}

POAG is a chronic degenerative disease characterized by damage to the optic nerve and affectation of the visual field. It is the second-leading cause of blindness worldwide, and the leading cause of irreversible blindness in adult populations [2]. The current objective of glaucoma treatment is to lower the rate of the loss of ganglion cells from the retina by reducing IOP [1]. Because POAG is a chronic progressive disease, its treatment must be long-term, even lifelong in some cases. Given this, it is important to balance the benefits of treatment with possible risks and adverse reactions. Also, in prolonged therapies tolerability is a key factor that can affect the efficacy of treatment due to its impact on adherence.

In controlled clinical trials with POAG patients, monotherapy with latanoprost at $0.005 \%$ reduced IOP levels to below $20-40 \%$ during a treatment period of 1-12 months [3]. Observations of patients treated for 24 months, meanwhile, showed that monotherapy with latanoprost reduced mean morning IOP both clinically and statistically, and that this reduction was maintained for up to 2 years of treatment. Finally, latanoprost has a good safety history [7].

The present study demonstrated that the preservative-free ophthalmic solution of $0.005 \%$ latanoprost (PRO-067) is effective in controlling IOP in POAG patients previously treated and controlled with GOF for at least 2 months. The transition from PRO-067 to GOF, and vice versa, after 30 days of use, did not affect the control of IOP in these POAG patients. In fact, non-inferiority was shown because the difference between the adjusted means of the two treatments was within the range of $-2.0 /+2.0 \mathrm{mmHg}$ postulated at the outset. The patients in sequence 1 experienced a reduction of $0.40 \mathrm{mmHg}$, while those in sequence 2 presented an increase of $0.10 \mathrm{mmHg}$ after using PRO-067. Neither value was significantly different, and did not reach a level that would indicate clinical importance. In fact, these findings are well below the non-inferiority limit of $2.0 \mathrm{mmHg}$.

Our results concur with earlier studies that found similar efficacy in reducing IOP using $0.005 \%$ latanoprost with BAK and BAK-free travoprost [8].

Currently, the efficacy and safety of GOF is well-known [6], but the preservative BAK included in its formulation has been related to a decrease in the stability of the tear film because it contains a compound of polyquaternary ammonium that affects the cell membranes and the lipid component of the tears, resulting in dry eyes and irritation [8, 13]. Preservative-free drugs can be useful in maintaining the integrity of the ocular surface, especially in glaucoma patients. It is estimated that approximately $50 \%$ of patients undergoing treatment present concomitant OSD. In addition, it is well-known that BAK-free PGAs present fewer toxic effects on the ocular surface $[8,14,15]$. Nevertheless, these preservative-free formulations must demonstrate their ability to maintain the efficacy described for their counterparts that contain a preservative $[12,15]$. PRO-067 (BAK-free latanoprost at $0.005 \%$ ) with integrated preservative-free system (low density polyethylene bottle, closure system of high density polyethylene with a silicone valve system), can be used for up to 48 months without refrigeration (store at $30^{\circ} \mathrm{C} \pm 2^{\circ} \mathrm{C}$ ). Eyes treated with preservative free medications also appear to have healthier ocular surfaces.

Our results demonstrate that the preservative-free ophthalmic solution of latanoprost at $0.005 \%$ (PRO-067) is just as effective in controlling IOP as the solution of latanoprost with BAK (GOF).

Another aspect concerns the identification of conjunctival hyperemia as the most common ocular complication associated with using PGAs $[12,3]$. This condition, however, is not an indicator of the toxicity of latanoprost but it is frequently due to a vasodilation effect $[6,18]$. Our results showed no significant differences in the presence or degree of hyperemia between the initial visit and after treatment with PRO-067. Moreover, the degree of severity of hyperemia decreased on the final visit in both sequences. Added to this, there were no statisticallysignificant differences between the sequences for TBUT, SFB, tearing or burning, and no observations of the presence of chemosis for either drug during the 8 weeks of the protocol.

As was expected, both latanoprost formulations proved to be safe, as there were no statistically-significant differences between PRO067 and GOF with respect to the incidence of adverse events related to the medications. Furthermore, most of the AEs observed were non-ocular in nature (75\%), and no serious adverse events occurred during the study.

The theoretical advantages of the BAK-free PRO-067 formulation for the ocular surface were not detected in this study, likely due to the short duration of the protocol (just 30 days). Earlier published studies have demonstrated that reductions in the frequency and severity of hyperemia can require 3-12 months to show significant differences between latanoprost with BAK and BAK-free treatments in POAG patients [12].

In other findings, the two formulations were similar in terms of the VF-14 index and the results of the questionnaire on ocular comfort. In general, both were well-tolerated. The VF-14 index was used to compare the impact of the two drugs on patients' daily lives, but there were no 
statistically-significant differences between the two groups in terms of quality of life related to the use of these medications. Furthermore, subjects reported few annoyances associated with their use, as reflected in their responses to the questionnaire on ocular comfort. Indeed, the only difference between the two sequences was an itching sensation that was not reported when using PRO-067.

Considering all these findings, we suggest that changing from GOF to PRO-067 maintains the effect of controlling IOP and could improve the comfort level of patients with respect to the ocular surface, since after 30 days of use the tolerability and safety of the two medications were similar.

\section{Conclusion}

In conclusion, PRO-067 is not inferior to GOF (GAAP Ofteno'), since observations showed control of the target IOP in patients who used PRO-067 compared to using GOF. Patients in sequence 1 presented a decrease of $0.40 \mathrm{mmHg}$, while those in sequence 2 experienced an increase of $0.10 \mathrm{mmHg}$ after using PRO-067. These differences are not statistically-significant and do not reach the level of clinical importance; indeed, they are well below the non-inferiority limit of $2.0 \mathrm{mmHg}$ posited at the outset of this study.

These results suggest, as well, that changing to preservative-free latanoprost at $0.005 \%$ is as effective as GOF, since after 30 days of use, the tolerability and safety of the two medications were similar, as were the results for ocular findings revealed by the VF-14 index, the questionnaire of ocular comfort, and the incidence of adverse events.

\section{Acknowledgments}

This study was supported by Laboratorios Sophia S.A. de C.V. (Zapopan, Jalisco, México). The authors thank Dávila J.F. (Servicios Médicos Quirúrgicos de Monterrey S.C.) and Stevenson M.O. (Hospital Fundación Santos y De la Garza Evia, I.B.P.). The study protocol was approved by their respective Institutional Review Boards, as follows: Comité de Ética en Investigación de Investigación Biomédica para el Desarrollo de Fármacos S.A. de C.V., Comité de Ética en Investigación del Hospital Hispano, S.A. de C.V., Comité de Ética en Investigación perteneciente a la Asociación para Evitar la Ceguera en México: APEC, Comité de Ética en Investigación de la Fundación de Asistencia Privada Conde de Valenciana I.A.P., and: Comité de Investigación de la Escuela de Medicina del Instituto Tecnológico y de Estudios Superiores de Monterrey.

The study was funded by Laboratorios Sophia, S.A. de C.V.

\section{Author Disclosure Statement}

\section{Conflicts of interest}

Leopoldo M. Baiza-Duran, Oscar Olvera-Montaño, Alejandra Sánchez-Rios and Patricia C. Muñoz-Villegas are employees of Laboratorios Sophia S.A. de C.V. The rest of authors declare that they have no competing interests.

\section{Authors' information}

The following institutions and researchers in Mexico participated in the study: Global Glaucoma Institute, Guadalajara, Jalisco; Unidad de Diagnóstico Temprano del Glaucoma, Zapopan, Jalisco; Instituto de Oftalmología y Ciencias Visuales, Universidad de Guadalajara, Guadalajara, Jalisco (Paczka, JA); Private Practice: F. Gómez Aguayo, Guadalajara, Jalisco (Gómez-Aguayo F); Asociación para Evitar la
Ceguera: APEC, Mexico City (Gil-Carrasco F); Aris Vision Institute, Guadalajara, Jalisco (Sánchez-Castellanos VE); Fundación de Asistencia Privada Conde de Valenciana I.A.P., Mexico City (Hartleben C); Servicios Médicos Quirúrgicos de Monterrey S.C., Monterrey, Nuevo Leon (Dávila JF) and, Fundación Santos y De la Garza Evia, I.B.P., Monterrey, Nuevo Leon (Stevenson MO). Baiza-Durán L, OlveraMontaño O, Sánchez-Rios A and Muñoz-Villegas P are employees of Laboratorios Sophia S.A. de C.V.

\section{Authors' contributions}

Sánchez-Castellanos VE, Paczka JA, Gómez-Aguayo F, GilCarrasco F and Hartleben C participated in data collection and study supervision. Paczka JA, Baiza-Durán L, Olvera-Montaño O, SánchezRios A and Muñoz-Villegas P participated in the study design, analysis and interpretation of data, drafting the manuscript, and its critical revision.

We thank the participants of the study.

\section{Data availability}

The datasets generated during and/or analyzed during the current study are available from the corresponding author on reasonable request.

\section{Registration of the study:}

ClinicalTrials.gov, NCT02801617.

\section{References}

1. Weinreb RN, Khaw PT (2004) Primary open-angle glaucoma. Lancet 363: 1711-1720. [Crossref]

2. Quigley HA, Broman AT (2006) The number of people with glaucoma worldwide in 2010 and 2020. Br J Ophthalmol 90: 262-267. [Crossref]

3. Perry CM, McGavin JK, Culy CR, Ibbotson T (2003) Latanoprost: an update of its use in glaucoma and ocular hypertension. Drugs Aging 20: 597-630. [Crossref]

4. Petounis A, Mylopoulos N, Kandarakis A, Andreanos D, Dimitrakoulias N (2001) Comparison of the additive intraocular pressure-lowering effect of latanoprost and dorzolamide when added to timolol in patients with open-angle glaucoma or ocular hypertension: a randomized, open-label, multicenter study in Greece. J Glaucoma 10 : 316-324. [Crossref]

5. van der Valk R, Webers CA, Schouten JS, Zeegers MP, Hendrikse F, et al. (2005) Intraocular pressure-lowering effects of all commonly used glaucoma drugs: a metaanalysis of randomized clinical trials. Ophthalmology 112: 1177-1185. [Crossref]

6. Gonzalez JR, Baiza-Duran L, Quintana-Hau J, Tornero-Montaño R, CastanedaHernandez G, et al. (2007) Comparison of the stability, efficacy, and adverse effect profile of the innovator $0.005 \%$ Latanoprost ophthalmic solution and a novel cyclodextrin-containing formulation. J Clin Pharmacol 47: 121-126. [Crossref]

7. Hedman K, Watson PG, Alm A (2002) The effect of latanoprost on intraocular pressure during 2 years of treatment. Surv Ophthalmol 47 Suppl 1: S65-76. [Crossref]

8. Rahmatnejad K, Rapuano CJ, Ichhpujani P, Wizov SS, Moster MR, et al. (2017) The Effects of Latanoprost With Benzalkonium Chloride Versus Travoprost With SofZia on the Ocular Surface. Eye Contact Lens. [Crossref]

9. Lee W, Lee S, Bae H, Kim CY, Seong GJ (2017) Efficacy and tolerability of preservative-free $0.0015 \%$ tafluprost in glaucoma patients: a prospective crossover study. BMC Ophthalmol 17: 61. [Crossref]

10. Servat JJ, Bernardino CR (2011) Effects of common topical antiglaucoma medications on the ocular surface, eyelids and periorbital tissue. Drugs Aging 28: 267-282. [Crossref]

11. Pisella PJ, Pouliquen P, Baudouin C (2002) Prevalence of ocular symptoms and signs with preserved and preservative free glaucoma medication. Br J Ophthalmol 86: 418423. [Crossref]

12. Aihara M, Otani S, Kozaki J, Unoki K, Takeuchi M, et al. (2012) Long-term effect of BAK-free Tavoprost on ocular surface and intraocular pressure in glaucoma patients after transition from Latanoprost. J Glaucoma 21: 60-64. [Crossref] 
Sánchez-Castellanos VE (2018) A phase III, randomized, clinical, crossover study to evaluate the non-inferiority of an ophthalmic solution of preservative-free latanoprost at $0.005 \%$ in patients with primary open-angle glaucoma

13. Anwar Z, Wellik SR, Galor A (2013) Glaucoma therapy and ocular surface disease: current literature and recommendations. Curr Opin Ophthalmol 24: 136-143. [Crossref]

14. Rasmussen CA, Kaufman PL, Kiland JA (2014) Benzalkonium chloride and glaucoma. J Ocul Pharmacol Ther 30: 163-169. [Crossref]

15. Goldberg I, Graham SL, Crowston JG, d'Mellow G; Australian and New Zealand Glaucoma Interest Group (2015) Clinical audit examining the impact the impact of benzalkonium chloride-free anti-glaucoma medications on patients with symptoms of ocular surface disease. Clin Experiment Ophthalmol 43: 241-220. [Crossref]
16. Stoney CM, Johnson LL (2012) Desing of clinical studies and trials. Principles and Practice of Clinical Research 225-242.

17. Anderson RD, Paterlla VM 1(999) Automated static perimetry, 2nd ed. St Louis: Mosby.

18. Thygesen J, Aaen K, Theodorsen F, Kessing SV, Prause JU (2000) Short-term effect of latanoprost and timolol eye drops on tear fluid and the ocular surface in patients with primary open-angle glaucoma and ocular hypertension. Acta Ophthalmol Scand 78: 37-44. [Crossref]

Copyright: C2018 Sánchez-Castellanos VE. This is an open-access article distributed under the terms of the Creative Commons Attribution License, which permits unrestricted use, distribution, and reproduction in any medium, provided the original author and source are credited. 